\title{
TESIS
}

Magíster de Ultrasonido en Cardiología. Facultad de Ciencias Médicas.
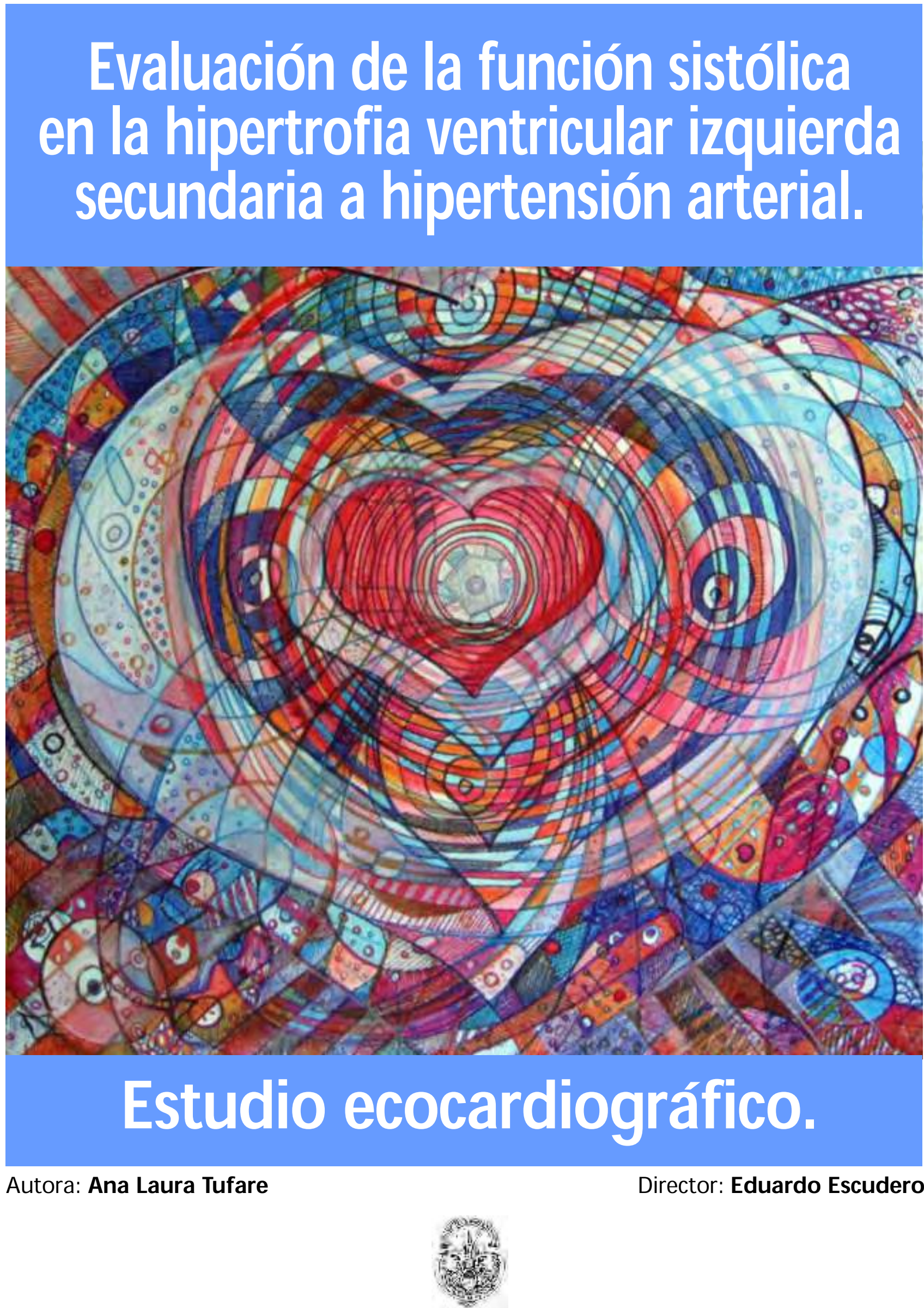


\title{
TESIS
}

Magíster de Ultrasonido en Cardiología. Facultad de Ciencias Médicas.

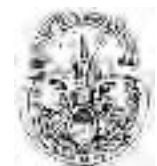

Universidad Nacional de La Plata

\section{EVALUACIÓN DE LA FUNCIÓN SISTÓLICA EN LA HIPERTROFIA VENTRICULAR IZQUIERDA SECUNDARIA A HIPERTENSIÓN ARTERIAL.}

\author{
ESTUDIO \\ ECOCARDIOGRÁFICO.
}


En la ciencia, la novedad surge sólo dificultosamente, manifestada por la resistencia, contra el fondo que proporciona lo esperado.

Inicialmente, sólo lo previsto y lo habitual se experimenta, incluso en circunstancias en las que más adelante podrá observarse la anomalía.

Sin embargo, un mayor conocimiento da como resultado la percepción de algo raro o relaciona el efecto con algo que se haya salido antes de lo usual.

Esta percepción de la anomalía abre un periodo en que se ajustan las categorías conceptuales, hasta que lo que era inicialmente anómalo se haya convertido en lo previsto.

En ese momento, se habrá completado el descubrimiento. 
ÍNDICE

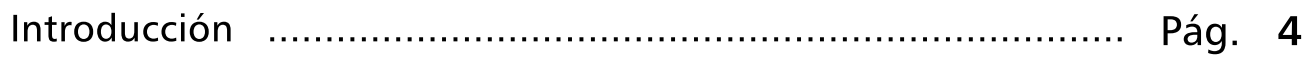

Hipótesis de Trabajo ....................................................... Pág. 7

Objetivo .................................................................. Pág. 7

Material y Métodos ...................................................... Pág. 8

Resultados ............................................................... Pág. 10

Discusión .................................................................. Pág. 18

Conclusión .............................................................. Pág. 20

Bibliografía ................................................................ Pág. 21

Í N D I C E D E T A B L A S Y F I G U R A S

Figura 1. Espesor Parietal ................................................. Pág. 4

Figura 2. Hipertrofia Ventricular Izquierda ............................. Pág. 6

Figura 3. Diámetros y espesores parietales en corte M del VI ..... Pág. 8

Tabla 1. Características demográficas de la población .............. Pág. 11

Tabla 2. Características ecocardiográficas de la población ........ Pág. 12

Figura 4. IMVI de las tres poblaciones estudiadas .................... Pág. 12

Figura 5. Espesor parietal relativo en los tres grupos de pacientes Pág. 13

Figura 6. EFS en los tres grupos analizados ............................. Pág. 13

Figura 7. Función sistólica del VI a través del \% de acortamiento medido a nivel endocárdico

Figura 8. Función sistólica del VI a través de \% de acortamiento medido a nivel de la mitad del espesor miocárdico

Pág. 14

Figura 9a. Regresión entre EFS y FAe analizada en la población control

Figura 9b. Regresión entre EFS y FAmv analizada en la población control Pág. 14

Figura 10. Porcentaje de acortamiento endocárdico Observado vs. Predecido expresado en \%

Pág. 15

Figura 11. Porcentaje de acortamiento medido a nivel de la parte media de la pared del miocardio Observado vs.

Predecido expresado en porcentaje

Pág. 15

Figura 12. Porcentaje de acortamiento medido a nivel de la parte media de la pared del miocardio Observado vs.

Predecido expresado en porcentaje, en donde en el grupo de HTA con HVI solo se agruparon los pacientes con H/R mayor a 0,47

Pág. 16

Tabla 3. Características demográficas de la población $\mathrm{HCH}$....... Pág. 16

Tabla 4. Características ecocardiográficas de la población HCH . Pág. 17 


\section{INTRODUCCIÓN}

La hipertrofia miocárdica ha sido clásicamente considerada como una respuesta adaptativa a largo plazo del músculo cardiaco ante condiciones de carga aumentada. Según la Ley de Laplace, el aumento del espesor de la pared produce una normalización del stress parietal, permitiendo una función cardiaca normal (figura 1) (1).

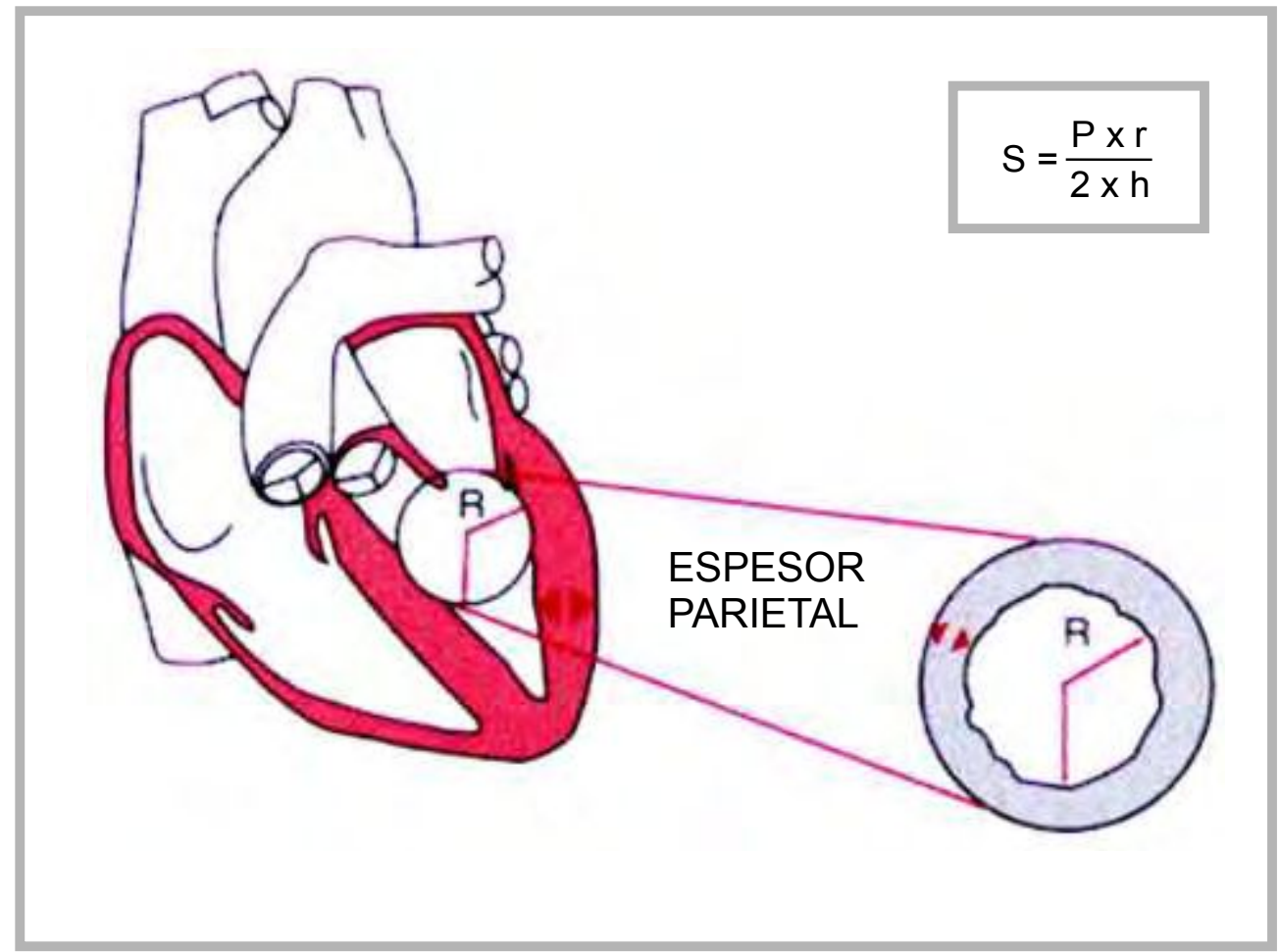

Figura 1

La sobrecarga de presión tiene un importante efecto sobre la síntesis de proteínas contráctiles y de matriz extracelular, a través del aumento de la proliferación de fibroblastos, del incremento de la expresión de genes de procolágeno por estos últimos y del aumento de la síntesis de proteínas (2). Debido a que estudios epidemiológicos han puesto en evidencia la asociación entre hipertrofia ventricular izquierda $(\mathrm{HVI})$ y aumento del riesgo de morbimortalidad cardíaca (3), se comenzó a cuestionar la hipótesis de que la hipertrofia es simplemente una respuesta adaptativa y surgió el concepto de la hipertrofia como una posible mala adaptación del corazón ante distintas situaciones patológicas. 
Existe una gran cantidad de estímulos que determinan el desarrollo de hipertrofia, cada uno de los cuales, activa señales moleculares, las cuales no sólo median el aumento de la masa miocárdica, sino que también son responsables de la activación de mecanismos deletéreos para el corazón, como por ejemplo la apoptosis, el estrés oxidativo y el deterioro de la función contráctil de los miocitos, con el consiguiente desarrollo de disfunción ventricular izquierda e insuficiencia cardiaca (4-7). Si una vía de señalización intracelular estimula tanto la hipertrofia como la muerte celular, la inhibición de dicha vía debería tener efectos beneficiosos. Por otro lado, si la activación de otra vía estimula el aumento de la masa miocárdica así como la sobrevida celular, la abolición de dicha señal, podría ser perjudicial, promoviendo la muerte celular a pesar de la reducción en la hipertrofia cardíaca.

Recientemente, Lu y col., publicaron evidencias acerca del efecto de la adenosina en los corazones de ratones sometidos a sobrecarga de presión. Por un lado, la adenosina brinda un efecto protector a través del receptor A1R, disminuyendo la hipertrofia, la fibrosis y la disfunción sistólica del VI. Por otro lado, los autores pusieron en evidencia el efecto opuesto cuando se evaluaba el efecto de la adenosina mediado por el receptor A3R (8).

Debido a la importancia de la HVI como factor de riesgo cardiovascular, deberían existir ciertos cambios específicos además de la masa por sí misma, que permitan predecir la futura transición de un estado de compensación a uno de descompensación, como podría ser el estado de la función ventricular.

Por lo tanto es bien conocido el deterioro de la función diastólica en este contexto, en donde la dinámica del llenado pasivo y la relación entre el volumen y la presión diastólica están influenciadas por la relajación activa y la deformación pasiva del miocardio, ambas directamente afectadas por el depósito exagerado de colágeno intersticial y la pérdida de la arquitectura miocárdica normal en los pacientes con HVI secundaria a sobrecarga de presión (9). Sin embargo existen marcadas controversias sobre el estado de la función sistólica del ventrículo izquierdo (VI) en las fases iniciales de la hipertensión arterial (HTA). Mientras que estudios experimentales empleando diferentes modelos de hipertensión señalan un deterioro de la contractilidad de la fibra hipertrófica aislada (10-14), observaciones en animales y en humanos sugieren que la misma está conservada, o incluso aumentada (15-17), utilizando a la ecocardiografía como herramienta de evaluación.

Por otro lado, clásicamente se ha utilizado el porcentaje de acortamiento medido a nivel del endocardio como índice de función sistólica, el cual ha demostrado no ser fidedigno en caso de aumento del espesor de la pared, Ilevando a sobreestimar la función sistólica (18-21) y arribar a conclusiones erróneas.

El análisis de la función en el corazón entero tomando la región medio- 
ventricular y no el endocardio como ha sido propuesto últimamente por varios autores (18-21) ha permitido llegar a resultados no tan contradictorios. Esto se debe a que si bien se asume que la mitad externa y la mitad interna del miocardio se engruesan en forma similar durante la sístole, el acortamiento en el subendocardio es mayor que en el subepicardio, lo cual se acentúa durante el desarrollo de hipertrofia ventricular, al aumentar el espesor de la pared $(19,22-26)$. Esto genera un desplazamiento mayor de la mitad interna de la pared del VI hacia el centro de la la cavidad que en un corazón no hipertrófico, manteniendo un acortamiento endocárdico normal a pesar de la disminución contráctil de las fibras aisladas. Estas observaciones se sumaron a razones anatómicas para considerar que el estudio de la función medio-ventricular podría representar con mayor exactitud el estado contráctil del miocardio. En efecto, las fibras orientadas preferentemente en sentido circunferencial predominan en la región medio ventricular, a diferencia que las subendocárdicas y subepicárdicas que están longitudinalmente orientadas (figura 2) (19).

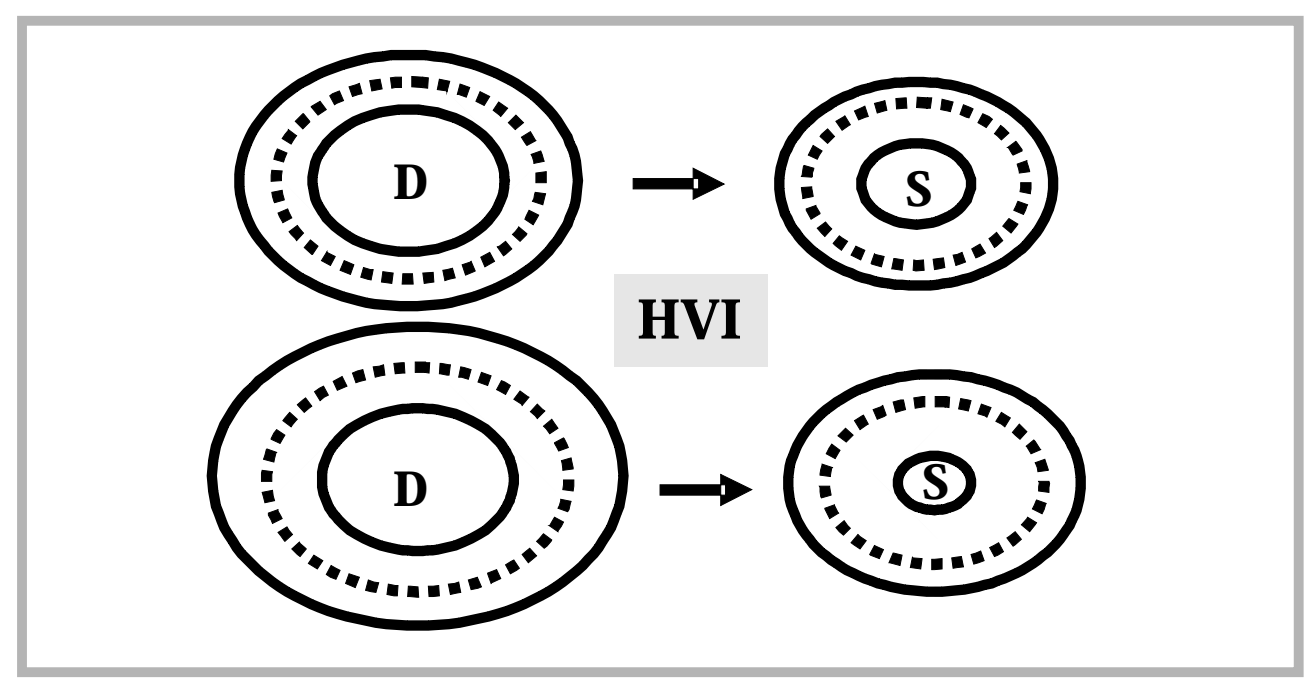

Figura 2

Diferentes autores han señalado la importancia del estudio del acortamiento en la porción medio-ventricular, señalando que en pacientes hipertensos con función sistólica normal a nivel endocárdico, la disminución del acortamiento a nivel de la mitad de la pared ventricular se asociaba a menor capacidad funcional (27) y/o a mayor aparición de eventos adversos (28).

Otro punto que debemos considerar es que tanto el porcentaje de acortamiento endocárdico (AE) como el medido a nivel de la parte media de la pared miocárdica constituyen índices sistólicos de fase eyectiva, por lo tanto 
ambos están afectados por las condiciones de carga del ventrículo izquierdo (29-32). En el contexto de la HVI secundaria a HTA, el engrosamiento parietal conlleva cambios importantes en el estrés de la pared (1). El grado de crecimiento de la pared no tiene una correlación lineal con los niveles de presión arterial, con lo que nos encontramos con individuos hipertensos que difieren notablemente en la respuesta hipertrófica y por lo tanto en el estrés parietal (33). Para realizar una correcta aproximación al estado contráctil del VI en un individuo determinado debemos considerar la poscarga que está afectando a ese ventrículo y analizar la función sistólica a través de la desviación en el acortamiento a partir del valor esperado para un nivel dado de estrés parietal sistólico (19). Esto nos permite obtener un índice independizado de la poscarga lo que nos posibilita realizar un análisis más acertado de la contractilidad cardíaca.

\section{HIPÓTESIS DE TRABAJO}

Si nos basamos en los resultados de estudios experimentales en donde la HVI secundaria a sobrecarga de presión determinó mayor progresión a insuficiencia cardíaca y muerte cardiovascular y en estudios en fibras miocárdicas aisladas de corazones hipertróficos donde se demostró deterioro de la contractilidad, deberíamos esperar que los pacientes hipertensos con HVI presenten alteraciones en la función contráctil desde etapas tempranas de la enfermedad.

El uso erróneo de índices ecocardiográficos poco sensibles y específicos para la evaluación de la función sistólica, junto con la falta de consideración del estado de poscarga en cada caso, podría ser la causa de los resultados contradictorios evidenciados en los estudios clínicos.

Por lo tanto, considero que este estudio permitirá aportar datos fidedignos acerca del estado de la función sistólica en la HVI secundaria a HTA.

\section{OBJETIVO}

El objetivo de este trabajo es comparar el estado de la función sistólica del VI en individuos hipertensos con y sin $\mathrm{HVI}$, determinando de esta forma si la respuesta hipertrófica es necesaria para mantener una función sistólica normal o si por lo contrario conlleva efectos deletéreos sobre la misma en el contexto de la HTA. 


\section{MATERIAL Y MÉTODOS}

Población: se incluyeron 72 individuos, 47 con diagnóstico de HTA, sin evidencias de otra comorbilidad cardiovascular (cardiopatía isquémica, valvulopatía, insuficiencia cardiaca, fibrilación auricular, etc.) ni diabetes, de los cuales 25 evidenciaban en el estudio ecocardiográfico un IMVI > a $125 \mathrm{~g} / \mathrm{m} 2$, conformando el grupo de pacientes hipertensos con $\mathrm{HVI}(\mathrm{HCH})$, y 22 con un IMVI < a $125 \mathrm{~g} / \mathrm{m} 2$, constituyendo el grupo de hipertensos sin HVI (HSH). El tercer grupo de individuos surgió de 25 pacientes sanos, denominándose grupo control (C).

A todos los individuos se les realizó un breve interrogatorio y examen clínico, para luego ser evaluados con un ecocardiograma.

Estudio ecocardiográfico: los estudios ecocardiográficos fueron realizados con un equipo ATL 3500 (Bothel Washington, ESA). Las distintas mediciones se realizaron según las guías de la American Society of Echocardiography (34). Se determinaron los diámetros y espesores parietales de fin de sístole y de fin de diástole en corte en Modo M del VI (fig. 3). Se calculó la masa ventricular izquierda (MVI), según la fórmula validada por Devereux y col. (33), en la que se le adjudica al VI una forma elíptica de configuración regular, con una relación entre la longitud del eje largo y del eje corto 2:1:

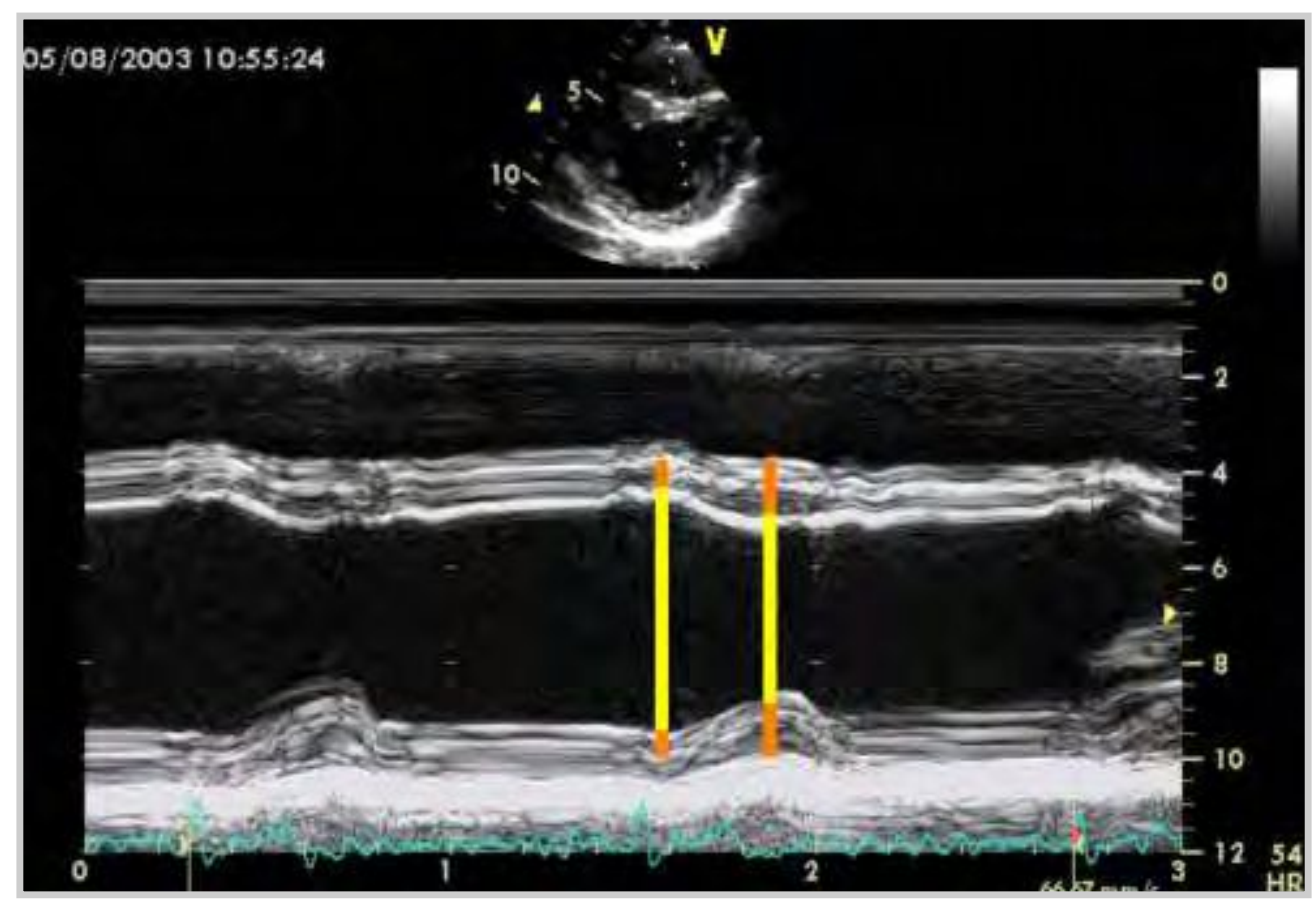

Figura 3 


$$
M V I=1.04((D D V I+S I V d+P P d) 3-D D V I 3)-13.6
$$

en donde DDVI es el diámetro de fin de diástole del VI, SIVd es el espesor en diástole del septum interventricular, PPd es el espesor en diástole de la pared posterior, 1.04 es el peso específico del miocardio y 13.6 corresponde a un factor de corrección. Para la determinación del índice de MVI (IMVI), se dividió la MVI por la superficie corporal del paciente. Se consideró como portadores de HVI aquellos individuos con valores de IMVI a $125 \mathrm{~g} / \mathrm{m} 2$ (36). El espesor parietal relativo $(\mathrm{h} / \mathrm{r})$ fue calculado con el fin de caracterizar la geometría del VI con la siguiente fórmula

$$
\mathrm{h} / \mathrm{r}=(\mathrm{SIVd}+\mathrm{PPd}) / \mathrm{DDVI}(37)
$$

Si bien existen recomendaciones recientes que sugieren realizar un punto de corte en 0.42 (38), se eligió utilizar la evaluación de la población control como parámetro de valores normales. Se definió como geometría concéntrica a aquellos valores de $h / r$ que superaban el valor promedio de $h / r$ más dos desvíos estándar del grupo control $(\mathrm{h} / \mathrm{r} \geq 0.47)$.

Para la evaluación funcional del VI se determinó la fracción de acortamiento endocárdica (FAe) y la fracción de acortamiento a nivel de la parte media del espesor de la pared ventricular (FAmv):

$$
\begin{gathered}
\mathrm{FAe}=[(\mathrm{DDVI}-\mathrm{DSVI}) / \mathrm{DDVI}]^{*} 100(34) \\
\mathrm{FAmv}=\frac{[(\mathrm{DDVI}+(\mathrm{TIVd}+\mathrm{PPd}) / 2)-(\mathrm{DSVI}+(\mathrm{TIVs}+\mathrm{PPs}) / 2)] * 100}{(\mathrm{DDVI}+(\mathrm{TIVd}+\mathrm{PPd}) / 2)}
\end{gathered}
$$

en donde DSVI corresponde al diámetro sistólico del VI, TIVs al espesor sistólico del tabique interventricular y PPs al espesor en sístole de la pared posterior.

Se calculó el estrés meridional de fin de sístole de la pared del VI para aproximarnos a la evaluación de la poscarga de dicho ventrículo:

$$
\mathrm{EFS}=\frac{0.334 \times \text { PAS } \times \text { DSVI }}{\text { PPs } x \quad[1+(\mathrm{PPS} / \mathrm{DSVI})]}(39)
$$

en donde PAS corresponde a la presión arterial sistólica obtenida en forma no invasiva al inicio del estudio.

Se examinaron además las relaciones entre la fracción de acortamiento endocárdico y del sector intermedio de la pared ventricular con el estrés telesistólico meridional. Para evaluar la función sistólica del VI independiente del estrés de fin de sístole, se calcularon las relaciones entre el acortamiento calculado a partir de las estimaciones ecocardiográficas y el esperado a partir 
del estrés telesistólico, empleando las ecuaciones que surgieron de la regresión entre el acortamiento tanto endocárdico como medioventricular y el estrés de fin de sístole de la población control.

Análisis estadístico: Los resultados fueron expresados como promedios \pm ES. Según correspondía, fueron utilizados para comparar las diferencias el test-t de Student o el análisis simple de varianza (ANOVA) con el StudentNewman-Keuls como post hoc test. La prueba estadística de chi-cuadrado se empleó para evaluar las diferencias de variables categóricas entre los grupos. La relación entre el acortamiento y el estrés de fin de sístole se analizó a través de una correlación lineal entre las variables.

Se consideró como significativas las diferencias con un valor de $p \leq a, 0,0$. 


\section{RESULTADOS:}

En la tabla I se enumeran las características demográficas de todos los individuos, en donde se observa que los pacientes hipertensos son mayores que los controles, siendo el grupo de $\mathrm{HCH}$ el de mayor edad. Los controles mostraron menor PA y mayor FC. El grupo de pacientes con HTA presentó BMI más alto. No se evidenciaron diferencias en el porcentaje de pacientes en tratamiento para la HTA ni en el tiempo transcurrido desde el diagnóstico entre los pacientes con y sin $\mathrm{HVI}$.

\begin{tabular}{|c|c|c|c|}
\hline $\begin{array}{l}\text { Tabla I } \\
\text { Características dem }\end{array}$ & gráficas de & la població & \\
\hline & C & HSH & $\mathrm{HCH}$ \\
\hline & $\mathrm{n}=\mathbf{2 5}$ & $\mathrm{N}=\mathbf{2 2}$ & $n=25$ \\
\hline edad (años) & $38.6 \pm 2.07^{*}$ & $57 \pm 3^{\star *}$ & $62.2 \pm 2.5$ \\
\hline sexo masc & $10(40 \%)$ & $9(40.9 \%)$ & $14(56 \%)$ \\
\hline BMI & $24.6 \pm 0.76^{*}$ & $28.3 \pm 1.2$ & $30.7 \pm 0.89$ \\
\hline TAS (mmHg) & $121 \pm 2,3^{*}$ & $146 \pm 3,1$ & $145 \pm 4.1$ \\
\hline $\mathrm{TAD}(\mathrm{mmHg})$ & $74,6 \pm 1,9^{*}$ & $91.5 \pm 1.7^{\star \star}$ & $86.4 \pm 2.2$ \\
\hline FC (Ixm) & $74 \pm 2.1^{\star *}$ & $73 \pm 2.6$ & $67 \pm 2.3$ \\
\hline Diag de HTA (años) & ........... & $10 \pm 2.8$ & $10.1 \pm 4.3$ \\
\hline Tto antihipert & - & $14(63 \%)$ & $20(74 \%)$ \\
\hline $\begin{array}{l}\text { BMI, indice de masa corpc } \\
\text { arterial diastólica; FC, frec }\end{array}$ & $\begin{array}{l}\text { al; TAS, presión } \\
\text { incia cardiaca. }\end{array}$ & otoria intóli & PAD, presión \\
\hline $\begin{array}{l}\text { "p<0.05 vs HTA } \sin H V I \\
\text { vs controles e HTA } \sin H 1\end{array}$ & TA con HVI: * & $<0.05$ vs HT & $\mathrm{HVl} ; \quad p<0,05$ \\
\hline
\end{tabular}

La tabla II resume las características ecocardiográficas de los tres grupos analizados, en donde los pacientes del grupo de $\mathrm{HCH}$ muestran aumento significativo del DDVI, de los espesores parietales, tanto en diástole como en sístole y de la MVI, siendo el IMVI mayor en el grupo de $\mathrm{HCH}$ con respecto a los $\mathrm{C}$ y a los $\mathrm{HSH}$, mostrando también diferencias significativas entre los $\mathrm{C}$ y los HSH (C: $88.7 \pm 3 \mathrm{~g} / \mathrm{m} 2 ; \mathrm{HSH}: 100 \pm 3 \mathrm{~g} / \mathrm{m} 2 ; \mathrm{HCH}: 161 \pm 5 \mathrm{~g} / \mathrm{m} 2, \mathrm{p} \leq 0.001$ ) (figura 4). 


\begin{tabular}{l} 
Tabla II \\
Caracteristicas ecocardiográficas de la población \\
\hline
\end{tabular}

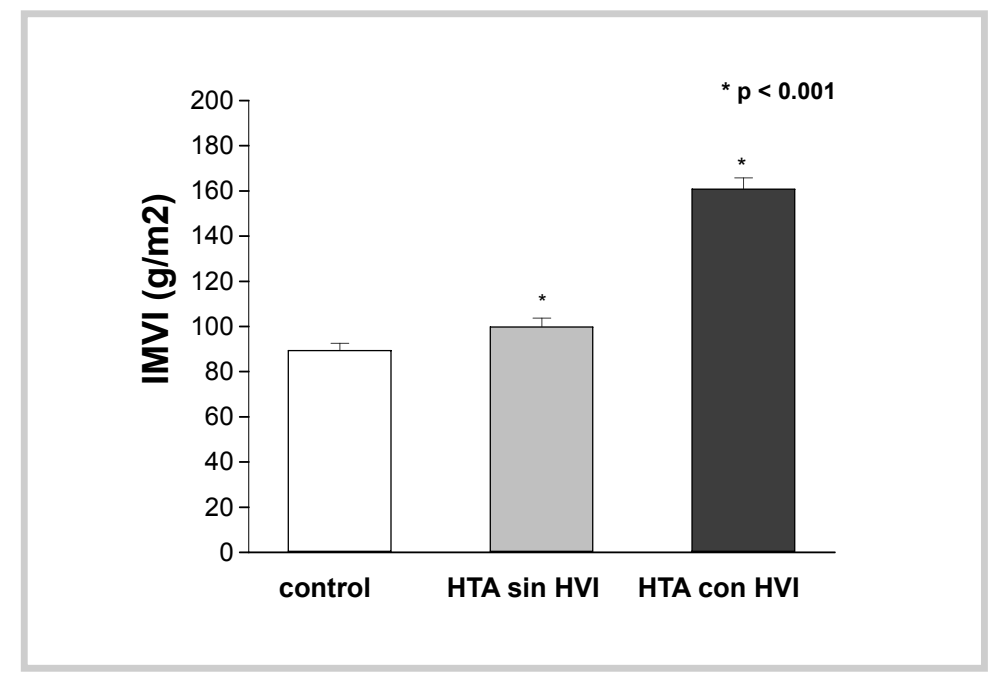

Figura 4: Las columnas representan el IMVI de las tres poblaciones estudiadas.

Al analizar la geometría ventricular observamos que a los cambios observados en el IMVI, se agrega un incremento del espesor parietal relativo en los pacientes con HTA, siendo el grupo de $\mathrm{HCH}$ el que presentó los valores más altos de $\mathrm{h} / \mathrm{r}$, objetivándose diferencias también significativas entre los $\mathrm{C}$ y lo $\mathrm{HSH}$ (C: $0,35 \pm 0,01 ; \mathrm{HSH}: 0,42 \pm 0,02 ; \mathrm{HCH}: 0,5 \pm 0,01, \mathrm{p}<0,01$ ) (figura 5). 


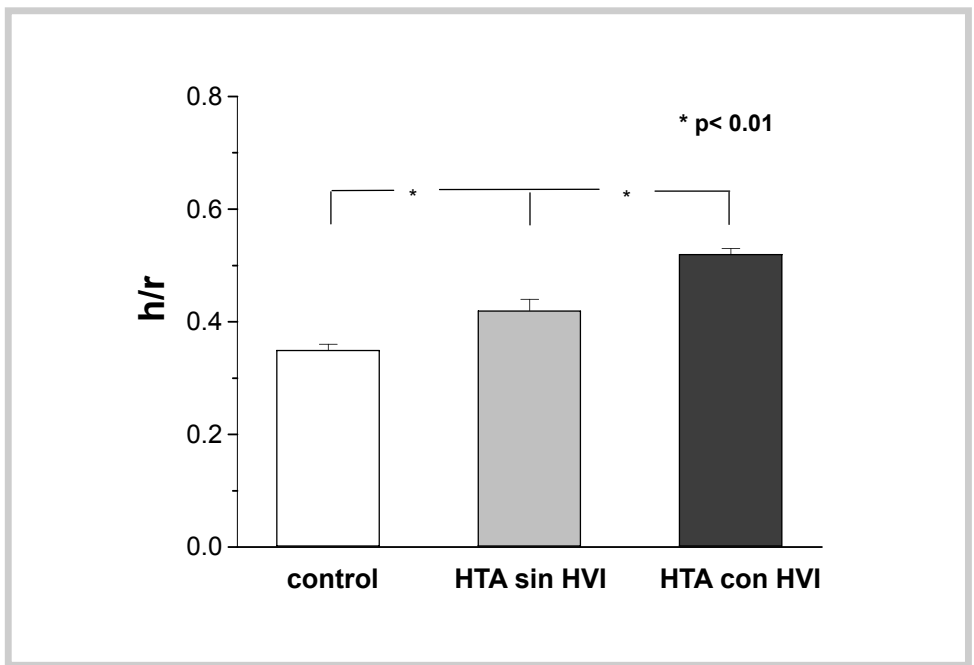

Figura 5: Se esquematiza el espesor parietal relativo en los tres grupos de pacientes.

Estos cambios, junto con los distintos niveles de PA, determinaron la presencia de diferencias en los valores de EFS, siendo menor en el grupo de $\mathrm{HCH}$ con respecto a los controles $(\mathrm{HCH}: 38,8 \pm 2$ kdynas/cm2; C: $51,2 \pm 2$ kdynas/cm2, $\mathrm{p}<0.01$ ) y existiendo una tendencia a ser menor en el grupo de $\mathrm{HSH}$ con respecto a los $\mathrm{C}$, sin llegar a ser significativa, no habiendo tampoco diferencias con respecto a los $\mathrm{HCH}$ (HSH: 43,1 23 kdynas/cm2, p ns) (figura 6).

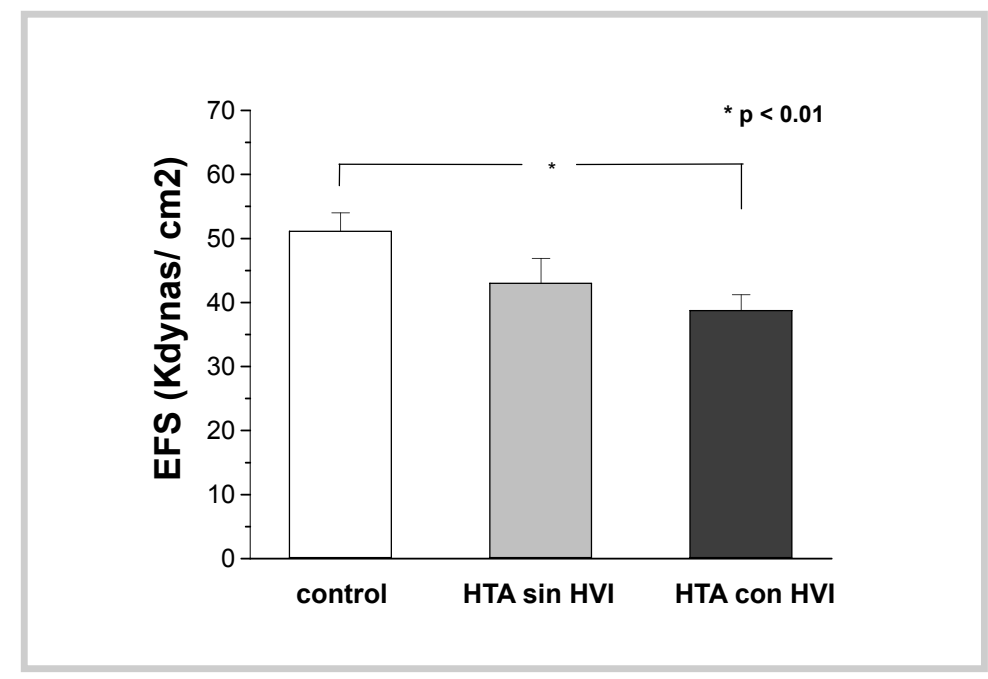

Figura 6: Se representa el EFS en los tres grupos analizados. 
Al analizar los parámetros de función sistólica del VI, observamos que, como ya había sido reportado en estudios previos (16-19), cuando el porcentaje de acortamiento se analiza a nivel del endocardio, en los grupos con alteración de la geometría ventricular (aumento del h/r), la FAe es mayor, siendo esta diferencia sólo estadísticamente significativa cuando se compara el grupo control con el grupo HSH (C: $39,4 \pm 1 \%$; $\mathrm{HSH}: 45,4 \pm 1,5 \%$; $\mathrm{HCH}: 43 \pm 1,7 \%$, p $<0.05$ ) (figura 7). Sin embargo esta diferencia no se manifiesta cuando el acortamiento se analiza a nivel de la parte media del espesor de la pared

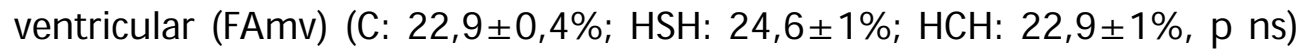
(figura 8).

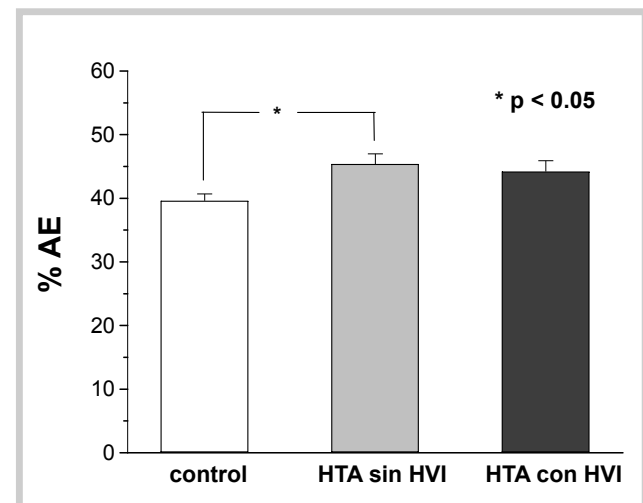

Figura 7: Función sistólica del VI analizada a través del porcentaje de acortamiento medido a nivel endocárdico.

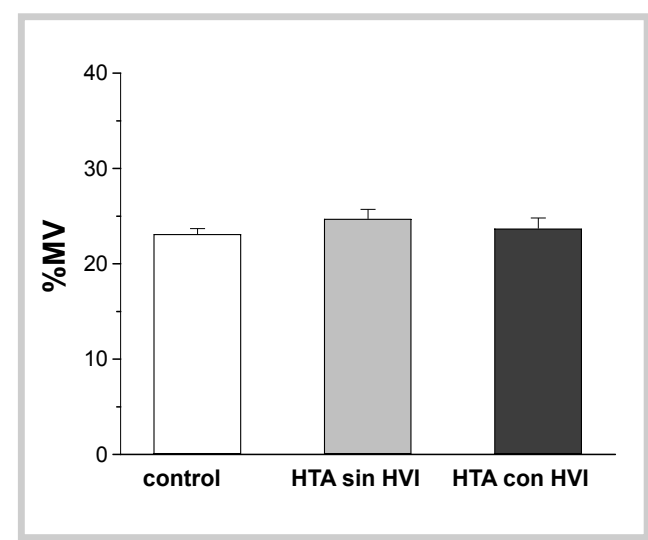

Figura 8: Función sistólica del VI analizada a través del porcentaje de acortamiento medido a nivel de la mitad del espesor miocárdico.

Teniendo en cuenta las diferencias encontradas en los niveles de PA y de geometría ventricular y por ende en el EFS entre los distintos grupos analizados, se evaluó la función sistólica independizada de la postcarga a través del índice que surge de la razón entre el porcentaje de acortamiento esperado (calculado a partir de la ecuación de la recta obtenida de la regresión lineal entre el acortamiento y el EFS de la población control, figuras 9A-9B) y el obtenido de los pacientes con HTA, expresado en porcentaje.

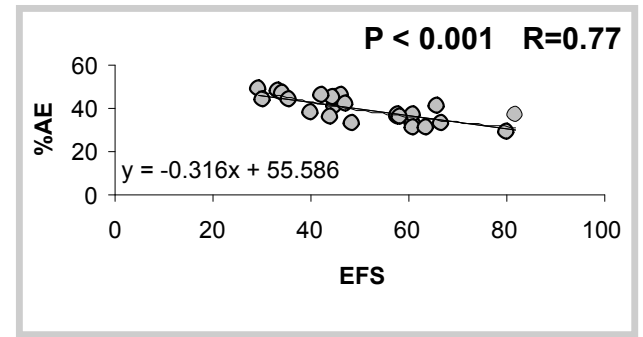

Figura 9a: Regresión entre EFS y FAe analizada en la población control.

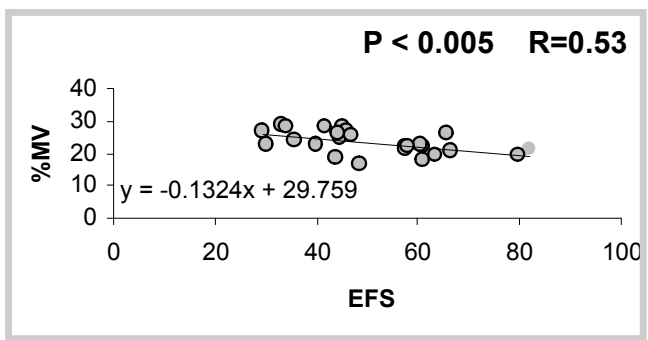

Figura 9b: Regresión entre EFS y FAmv analizada en la población control. 
Cuando se analizó el índice a nivel del endocardio, se evidenciaron valores significativamente más altos en el grupo $\mathrm{HSH}$ con respecto tanto a los $\mathrm{C}$ y al grupo de $\mathrm{HCH}$, sin diferencias entre estos dos grupos (C: 100,2 $2 \% ; \mathrm{HCH}$ : $98,6 \pm 2,5 \%$ vs $\mathrm{HSH}: 108,5 \pm 2,4 \%$ p 0,01 ) (figura 10 ), lo cual se debería interpretar teniendo en cuenta que la medida a nivel del endocardio implica una sobreestimación en los grupos con incremento del espesor parietal relativo.

Teniendo en cuenta este último concepto los valores similares del grupo de $\mathrm{HCH}$ con los $\mathrm{C}$ estarían indicando un deterioro de este parámetro en los pacientes portadores de HVI. Esto se pone de evidencia al analizar el índice tomando el acortamiento medido a nivel de la mitad de la pared miocárdica en dónde desaparecen las diferencias a favor del grupo de $\mathrm{HSH}$, existiendo sólo una tendencia de valores más bajos en el grupo de $\mathrm{HCH}$ (C: 100,3 $\pm 2,5 \%$;

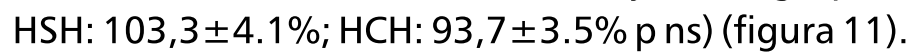

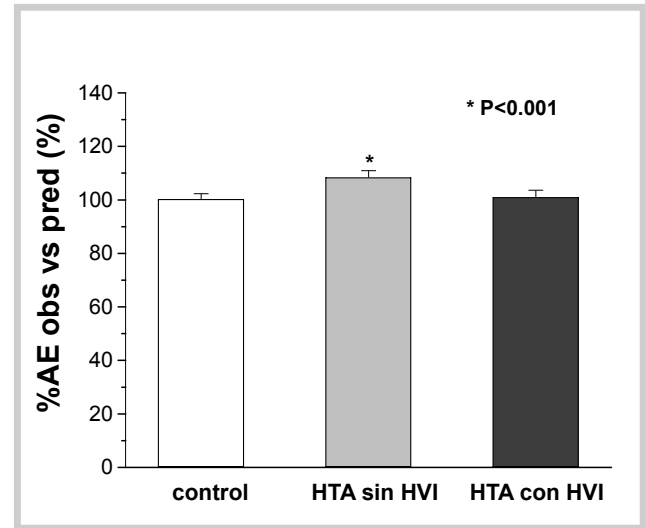

Figura 10: Porcentaje de acortamiento endocárdico observado vs predecido expresado en porcentaje.

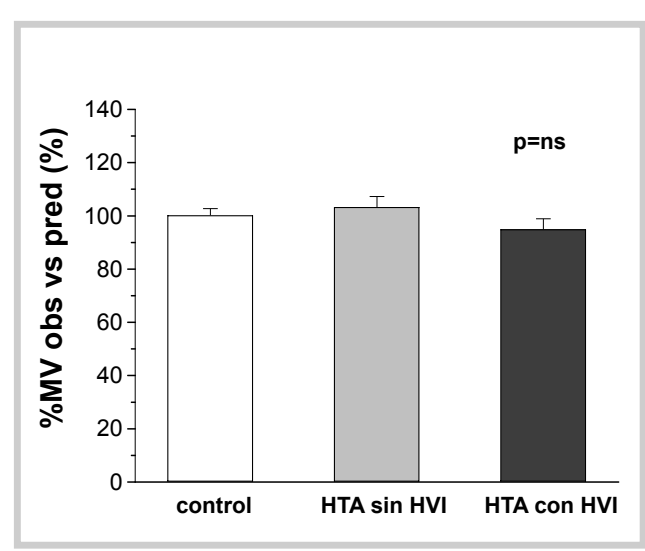

Figura 11: Porcentaje de acortamiento medido a nivel de la parte media de la pared del miocardio observado vs predecido expresado en porcentaje.

Si bien no se objetivaron diferencias significativas en el análisis del porcentaje de acortamiento medido a nivel de la parte media de la pared del miocardio independizado de la postcarga (\%MV obs vs pred) entre los grupos en estudio, se observó una clara tendencia de valores inferiores en la población hipertensa con HVI. Al analizar sólo los pacientes con HVI con espesor parietal relativo $\geq$ al valor promedio \pm 2 DS de la población control $(h / r \geq a 0,47)$ y compararlos con los $\mathrm{C}$ y los $\mathrm{HSH}$, se pone en evidencia una significativa disminución de este índice en los pacientes con $\mathrm{HVI}$ concéntrica ( $\mathrm{C}$ : $100,3 \pm 2,5 \%$; $\mathrm{HSH}: 103,3 \pm 4.1 \%$ vs $\mathrm{HCH}: 90.9 \pm 4 \% \mathrm{p}<0.05$ ) (figura 12 ). 


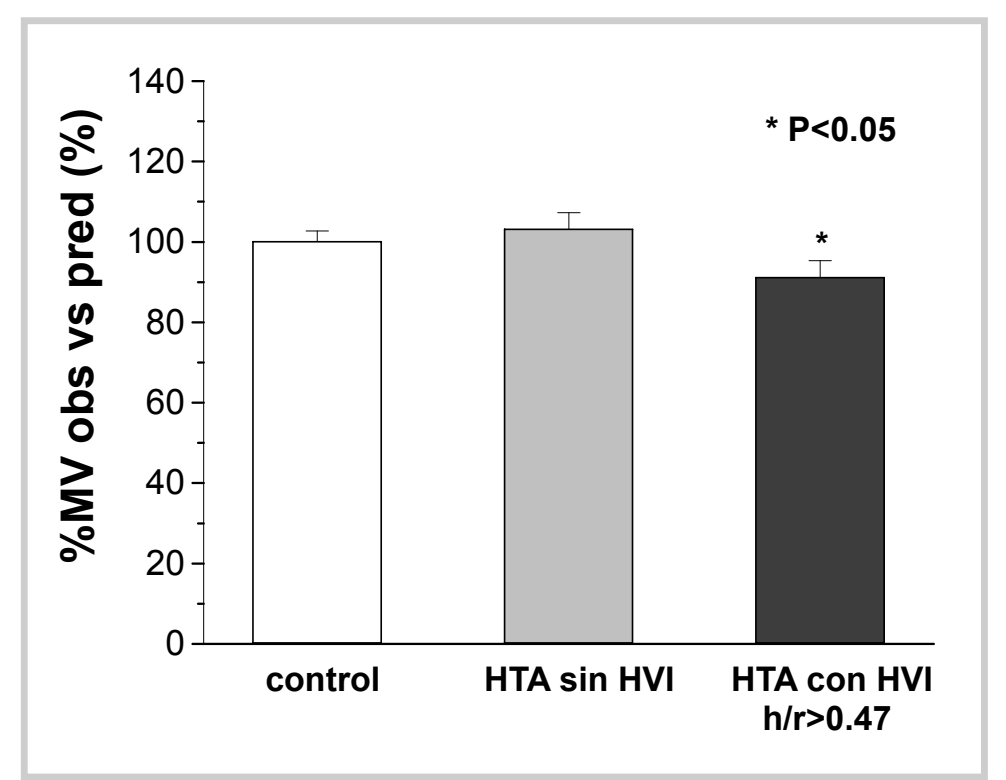

Figura 12: Porcentaje de acortamiento medido a nivel de la parte media de la pared del miocardio observado vs predecido expresado en porcentaje, en dónde en el grupo de HTA con HVI solo se agruparon los pacientes con $h / r>$ a 0.47 .

No se evidenciaron diferencias estadísticamente significativas al comparar las características demográficas en la población portadora de HVI al dividirla en los subgrupos según $\mathrm{h} / \mathrm{r}>0<\mathrm{a} 0.47$ (tablas III). Al comparar los datos ecocardiográficos, sólo se objetivaron diferencias significativas en el diámetro diastólico del $\mathrm{VI}$, siendo mayor en los pacientes con $\mathrm{h} / \mathrm{r}<\mathrm{a} 0.47$ y en los espesores del septum interventricular y la pared posterior en diástole con un incremento en el grupo con HVI concéntrica (tabla IV).

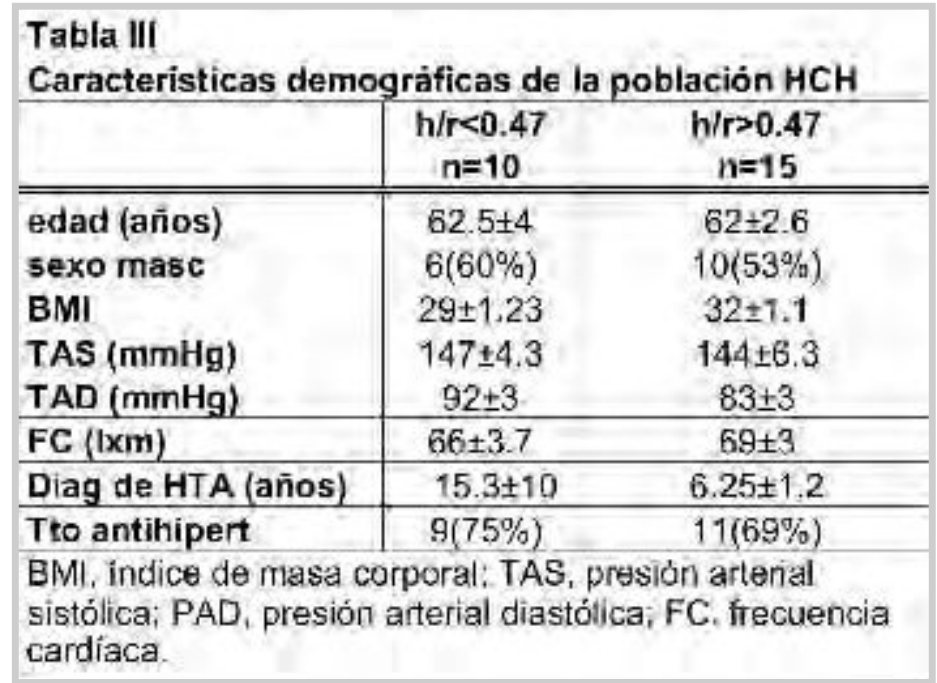




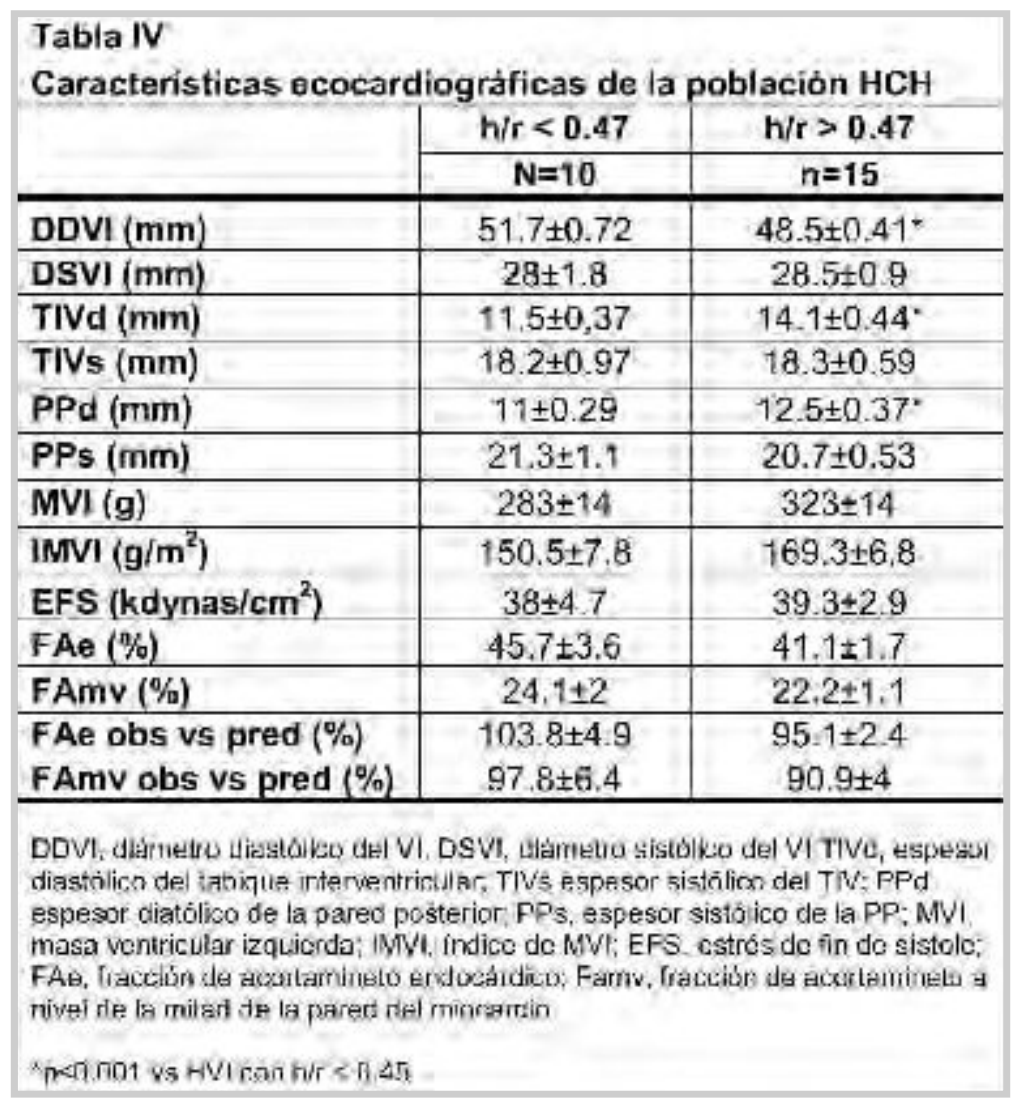




\section{DISCUSIÓN:}

Los resultados de este estudio demuestran que los pacientes hipertensos con HVI y alteración de la geometría ventricular presentan deterioro en la función sistólica no evidenciada con los índices ecocardiográficos clásicos. Esto se objetiva cuando se tiene en cuenta en el análisis la poscarga y se utiliza un parámetro ecocardiográfico específico y sensible como lo es el porcentaje de acortamiento medioventricular. De esta forma se realiza una evaluación fisiopatológica adecuada, sin sobreestimación de la función sistólica como suce-de en los estudios en los que se utiliza tanto el porcentaje de acortamiento endocárdico como la fracción de eyección y además se independiza la fun-ción sistólica del estado de poscarga, acercándonos a la estimación de la contractilidad, objetivo más preciado en la evaluación de la función sistólica del VI. Al referirnos a los datos provenientes de la bibliografía encontramos información controvertida. Cuando analizamos los estudios en animales, en donde existe la posibilidad de investigar la contractilidad en forma más directa a través de herramientas invasivas como las curvas de presión volumen o la contractilidad de las fibras musculares aisladas, la discrepancia es menor. Por un lado, Megure et al. (40), estudiaron los efectos de la inhibición de la calcineurina, una fosfatasa activada por calcio/calmodulina y responsable del desarrollo de hipertrofia, por ciclosporina A en modelos de ratones con sobrecarga de presión, demostrando que la disminución de la respuesta hipertrófica estaba asociada a un incremento significativo de la mortalidad debido a insuficiencia cardíaca. Estos resultados avalarían el concepto de que la $\mathrm{HVI}$ es un mecanismo compensador beneficioso que protege al corazón en presencia de sobrecarga de presión. Sin embargo, mas recientemente, Hill et al. (41), reportaron resultados totalmente opuestos, demostrando en su experiencia, que la inhibición de la hipertrofia por ciclosporina A no produjo aumento del tamaño del corazón ni deterioro de la función ventricular a pesar de la sobrecarga de presión, poniendo en duda la hipótesis anteriormente mencionada y argumentando el hecho de que la HVI no sería indispensable para la adaptación del corazón al aumento de las cargas y que en realidad sería una "mala" adaptación en esta situación.

A favor de esto último, datos publicados por Esposito el al. (42), demuestran que en dos modelos de ratones transgénicos con una respuesta hipertrófica abolida, uno a través de la expresión cardíaca del péptido carboxilo terminal $\mathrm{Gq}$, que específicamente inhibe las señales mediadas por $\mathrm{Gq}$, y otro modelo con deficiencia en el gen de Dopamina B-hidroxilasa, lo cual determina una depleción endógena de epinefrina y norepinefrina, a pesar de que el stress parietal permaneció aumentado, la función cardíaca mostró menor deterioro que el sufrido por los ratones con respuesta hipertrófica conservada y stress parietal normal. 
Al referirnos a los estudios clínicos sobre HVI, vemos que a pesar de informar que la función sistólica está conservada o incluso aumentada con respecto a controles, se objetiva un peor pronóstico en estos pacientes con una mayor progresión a insuficiencia cardíaca, constituyendo la HVI un factor de riesgo independiente de morbimortalidad cardíaca en la HTA. Por otro lado, al evaluar los efectos de distintas drogas antihipertensivas sobre la estructura y función del VI, se deja en evidencia que la regresión de la HVI conlleva una mejoría en la función sistólica del VI y en el pronóstico clínico a largo plazo (43).

Debemos tener en cuenta que el deterioro contráctil encontrado en las miofibrillas en un miocardio hipertrófico puede generar una fracción de eyección normal, debido a la reduplicación de sarcómeros dispuestos en paralelo que compensaría la disminución contráctil referida (22). La contracción sub-normal de los sarcómeros extras en paralelo lleva a mantener similar engrosamiento y similar desplazamiento de sangre a los que se tenía con un menor número de sarcómeros pero con mejor función. Por lo tanto es importante tener en cuenta que una fracción de eyección de 0.55 en un ventrículo con hipertrofia concéntrica estaría indicando una disminución de la función sistólica $(44,45)$.

Esto se pone en evidencia en los resultados de este estudio, en donde el análisis de la función sistólica a través de la fracción de acortamiento endocárdico revela un incremento en los valores en pacientes con HSH y valores similares a los controles en pacientes con $\mathrm{HCH}$, lo que haciendo un análisis fisiopatológico adecuado estaría indicando una similar función en los pacientes HSH con los controles, en los cuales la sobrevaloración estaría determinada por la alteración de la geometría ventricular (aumento del espesor parietal relativo con respecto a los controles) y una disminución en el estrés de fin de sístoles de la pared ventricular (disminución de la poscarga). Los valores similares entre los controles y los pacientes del grupo $\mathrm{HCH}$ indicaría, bajo este mismo análisis interpretativo, un deterioro en la función sistólica (los pacientes con HVI tienen mayor número de miofibrillas contrayéndose y el VI está sometido a menor estrés parietal debido a un incremento significativo del espesor de la pared con respecto al diámetro del $\mathrm{VI})$.

En una experiencia previa, en donde estudiamos la función sistólica en pacientes con $\mathrm{HVI}$ secundaria a HTA y deportistas con HVI, a través del análisis de la velocidad de deformación de un segmento de la pared ventricular (strain rate), herramienta ecocardiográfica independiente de la poscarga de reciente desarrollo, llegamos a resultados concordantes. Los pacientes con HVI secundaria a HTA presentaban valores significativamente menores comparada con el grupo control y con los deportistas (46).

Estos hallazgos coinciden con datos publicados recientemente por Strotman 
y col., quienes evaluaron la función sistólica a través del strain rate en pacientes hipertensos y en pacientes portadores de estenosis aórtica severa. Al compararlos con controles sanos objetivaron valores de strain rate sistólico significativamente más bajos en los grupos de pacientes con HVI (47).

Este estudio también pone en evidencia que la falta de detección de deterioro de la contractilidad en etapas tempranas de la HVI no se debería a una limitación en los índices ecocardiográficos clásicamente usados, sino a una errónea interpretación de los datos hallados. Esto implica que con herramientas sencillas y disponibles en todos los laboratorios de ecocardiografía como lo es la obtención de un corte en Modo M del VI y el registro de la PA sistólica, se puede lograr una correcta evaluación de la función sistólica en pacientes con $\mathrm{HVI}$, sin requerir para esto tecnología cara y de difícil disponibilidad.

\section{CONCLUSIÓN:}

Según los resultados de este estudio, los pacientes hipertensos que presentan alteración estructural del VI del tipo HVI concéntrica, presentan alteración de la función sistólica evidenciada al considerar en su análisis un índice específico como lo es la fracción de acortamiento medioventricular independizado del estado de poscarga. 


\section{BIBLIOGRAFÍA:}

1. Sadoshima J, Izumo S. The cellular and molecular response of cardiac myocytes to mechanical stress. Annu Rev Physiol. 1977; 59: 551-557.

2. Bishop J, Lindahl G. Regulation of cardiovascular collagen synthesis by mechanical load. Cardiovas Res 1999; 42: 27-44

3. Kannel WB, Gordon T, Offut D Left ventricular hypertrophy by electrocardiogram: prevalence, incidence and mortalitiy in the Framingham Study. Ann Intern Med 71: 89-105, 1969.

4. Morisco C, Sadoshima J, Trimarco B, Arora R, Vatner D, Vatner S. Is treating cardiac hypertrobhy salutary or detrimental: the two faces of Janus. Am J Physiol Heart Circ Physiol. 284: H1043-H1047. 2003.

5. Motoaki S, Schneider M. Still Stressed Out but Doing Fine. Normalization of wall stress is superfluous to mainteining cardiac function in chronic pressure overload. Circulation 2002; 105:8-10.

6. Condorelli G, Morisco C, Stassi G, Notte A, Farina F, Sgaramella G, de Rienzo A, Roncarati R, Trimarco B, Lembo G. Increased cardiomyocyte apoptosis and changes in proapoptotic and antiapoptotic genes bax and bcl-2 durin left ventricular adaptations to chronic pressure overload in the rat. Circulation 99: 3071-3078. 1999.

7. Lu Z, Xu X, Hu X, Zhu G, Zhang P, van Deel E, French J, Fasset J, Oury T, Bache R, Chen Y. Extracelular superoxide dismutase deficiency exacerbates pressure overload-induced left ventricular hypertrophy and dysfunction. Hypertension 2008; 51: 19-25.

8. Lu Z, Fasset J, Xu X, Hu X, Zhu G, French J, Zhang P, Schnermann J, Bache R, Chen Y. Adenosine A3 receptor deficiency exerts unanticipated protective effects on the pressure-overload left ventricle. Circulation 2008; 118: 1713-1721.

9. Brutsaer DL, Sys SU, Gillebert TH. Diastolic failure. Pathophysiology and therapeutic implications. J Am Coll cardiol 1993; 22: 318-325.

10. Minamisawa S, Hoshijima M, Chu G. Chronic phospholamban-sarcoplasmic reticulum calcium ATPase interaction is the critical calcium cycling defect in dilated cardiomyopathy. Cell. 1999; 99: 313-322.

11. Shan K, Bick RJ, Poindexter. Relation of tissue Doppler derived myocardial velocities to myocrdial structure and beta adrenergic receptor in humans. J Am Coll Cardiol. 2000; 36: 891896.

12. Bing $\mathrm{OHL}$, Matsushita $\mathrm{S}$, Fanburg BL, Levine BL, Levine HJ. Mechanical properties of rat cardiac muscle during experimental hypertrophy. Circ Res. 1971; 28: $234-245$.

13. Alpert NR, Hamrell BB, Halpern W. Mechanical and biochemical correlates of cardiac hypertrophy. Circ Res. 1974; 34/35(suppl II): II-71-II-82.

14. Hamrell BB, Hultgren PB. Sarcomere shortening in pressure overload hypertrophy. Fed Proc. 1986; 45: 2591-2596.

15. De Simone G, Di Lorenzo L, Constantino G. Moccia D, Buoníssimo S, de Divitiis O: Supernormal contractility in primary hypertension without left ventricular hypertrophy. Hipertensión Dallas 1988; 11: 457- 63.

16. Van Hooft IMS, Grobbee DE, Waal-Manning HJ: Hemodynamic characteristics of the early phase of primary hypertension. The Dutch hypertension and offspring study. Circulation 1993; 87: 1100-06.

17. De Simone G, Devereux RB, Volpe M, Camargo MJF, Wallerson WC, Laragh JL: Relation of left ventricular hypertrophy afterload and contractility to left ventricular performance in Goldblatt hypertension. Am J Hypertens 1992; 5: 292-01.

18. Shimuzu G, Hirota Y, Kita Y, Kawamura K, Saito T,Gaasch W: Left ventricular midwall mechanics in systemic arterial hypertension. Myocardial function is depressed in pressure-overloaded hypertrophy. Circulation 1991; 83: 1676-84.

19. De Simone G, Devereux RB, Roman MJ, Ganau A, Saba PS, Alderman MH, et al: Assessment of left ventricular function by the midwall fractional shortening-end-systolic stress relation in human hypertension. J Am Coll Cardiol 1994; 23: 1444-51. 
20. De Simone G, Devereux RB, Vople M, Camargo MJF, Wallerson DC, Laragh J: Midwall LV mechanics in rats with or without renovascular hypertension: effect of different $\mathrm{Na}+$ intakes. Am J Physiol 1996; 270: H628-37.

21. Escudero E, Pérez G, Camilión de Hurtado C, Tufare A. Echocardiographic assessment of left ventricular midwall mechanics in spontaneously hypertensive rats. Eur J Echocardiography 2004; 5: 169-175.

22. Shimizu G, Zile MR, Blaustein AS, et al. Left ventricular chamber filling and mid-wall fiber lengthening in patients with left ventricular hypertrophy: overestimation of fiber velocities by conventional midwall measurements. Circulation. 1985; 71: 266-272.

23. Palmon $L$, Reichek N, Yeon S, Clark N, Brownson D, Hoffman E, Axel L. Intramural myocardial shortening in hypertensive left ventricular hypertrophy with normal pump function.

24. Circulation. 1994; 89: 122-131.

Aurigemma GP, Gaasch WH, McLaughlin M, McGinn R, Sweeney A, Meyer TE. Reduced systolic pump performance and depressed myocardial contractile function in patients with normal ejection fraction and high relative wall thickness. Am J Cardiol. 1995; 76: 702-705.

25. Aurigemma GP, Silver KH, Priest MA, Gaasch WH. Geometric changes allow normal ejection fraction despite depressed myocardial shortening in hypertensive left ventricular hypertrophy. J Am Coll Cardiol. 1995; 26: 195-202.

26. Sadler DB, Aurigemma GP, Williams DW, Reda DJ, Materson BJ, Gottdiener JS. Systolic function in hypertensive men with concentric remodelling. Hypertension. 1997; 30: 777-781.

27. Schussheim AE, Devereux RB, de Simone G, Borer JS, Herrold EMcM, Laragh JH. Usefulness of subnormal midwall fractional shortening in predicting left ventricular exercise dysfunction in asymptomatic patients with systemic hypertension. Am J Cardiol. 1997; 79: 1070-1074.

28. de Simone G, Devereux RB, Koren MJ, Mensah GA, Casale PN, Larga JH. Midwall left ventricular mechanics: an independent predictor of cardiovascular risk in arterial hypertension. Circulation. 1996; 93: 259-265.

29. Mahler F, Ross J Jr, O'Rourke RA, et al. Effects of changes in preload, afterload and inotropic state of ejection and isovolumic phase measures of contractility in the conscious dog. Am J Cardiol. 1975; 35: 625-634.

30. Ross J Jr. Afterload mismatch and preload reserve: a conceptual framework for the analysis of ventricular function. Prog Cardiovasc Dis. 1976; 18: 255-264.

31 Borrow KM, Neumann A, Marcus RH, et al. Effects of simultaneous alterations in preload and afterload on measurements of left ventricular contractility in patients with dilated cardiomyopathy: comparisons of ejection phase, isovolumetric and end-systolic force-velocity indexes.J Am Coll Cardiol. 1992; 20: 787-795.

32. Kreulen TH, Bove AA, McDonough MT, et al. The evaluation of left ventricular function in man: a comparison of methods. Circulation. 1975; 51: 677-688.

33. Drayer JI, Gardin JM, Brewer DD, Weber MA. Disparate relationships between blood pressure and left ventricular mass in patients with and without left ventricular hypertrophy. Hypertension 1987; 9 (Suppl II): II61-II64.

34. Sahn DJ, De María A, Kisslo J, Weyman A. The Committee on M-mode Standarization of the American Society of Echocardiography: recommendations regarding quantitation in M-mode echocardiography. Results of a survey echocardiographic measurements. Circulation 1978; 58: 1072-83

35. Devereux R, Alonso D, Lutas E, Goettlieb G, Campo E, Sachs I, Reichek N. Echocardiographic assessment of left ventricular hypertrophy: Comparision to necropsy findings. Am J Cardiol 1986; 57: 450-458.

36. Devereux R, Reichek N. Echocardiographic determination of left ventricular mass in man: anatomic validation of the method. Circulation 1977; 55:613-618.

37. Gaasch WH. Left ventricular radius to wall thickness ratio. Am J Cardiol 1979; 43: 1189-1194.

38. Lang R, Bierig M, Devereux R, Flachskampf F, Foster E, Pellikka P, Picard M, Roman M, Seward J, Shanewise J, Solomon S, Spencer K, St John Sutton M, Stewart W and Members of the Chamber Quantification Writing Group. Recommendations for Chamber Quantification: A 
Report from the American Society of Echocardiography's Guidelines and Standards Committee and the Chamber Quantification Writing Group, Developed in Conjunction with the European Association of Echocardiography, a Branch of the European Society of Cardiology. JASE 2005; 18 (12): 1440-1463.

39. Grossman W, Jones D, McLaurin L. Wall stress and patterns of hypertrophy in the human left ventricle. J Clin Invest 1975; 56: 56-64.

40. Meguro T, Hong C, Asai K, Takagi G, McKinsey TA, Olson EN, Vatner SF. Cyclosporine attenuates pressure-overload hypertrophy in mice while enhancing susceptibility to descompensation and heart failure. Circ Res 84: 735-740. 1999.

41. Hill JA, Karimi M, Kutschke W, Davisson RL, Zimmerman K, Wang Z, Kerber RE, Weiss RM. Cardiac hypertrophy is not a required compensatory response to short term pressureoverload. Circulation 101: 2863-2869. 2000.

42. Esposito G, Paracciuolo A, Naga Prasad SV, Takaoka H, Thomas SA, Koch WJ, Rockman HA. Genetic alterations that inhibit in vivo pressure-overload hypertrophy prevent cardiac dysfunction despite increasd wall stress. Circulation 105: 85-92. 2002.

43. Kjelden SE, Dahlof B, Devereux RB, et al. Effects of losartan on cardiovascular morbidity and mortality in patients with isolated systolic hypertension and left ventricular hypertrophy: a Losartan Intervention for Endpoint Reduction (LIFE) substudy. JAMA 288: 1491-1498. 2002.

44. De Simone G, Devereux RB, Celentano A, et al. Left ventricular chamber and wall mechanics in the presence of concentric geometry. J Hypertens. 1999; 17: 1001-1006.

45. Carabello B. Evolution of the study of left ventricular function. Everithing old is new again. Circulation 2002; 105: 2701-2703.

46. Tufare AL, Escudero EM, Ankudowicz V. Deterioro temprano de la contractilidad en le hipertrofia secundaria a hipertensión. Revista FAC 2005; 34 (Supl. II): 53).

47. Strotman J, Lengenfelder B, Blondelot J, Voelker W, Herrman S, Ertl G, Weidermann F. Functional differences of left ventricular hypertrophy induced by either arterial hypertension or aortica valve stenosis. Am J Cardiol. 2008; 101: 1493-1497. 